\title{
To recovering of continuous function by sequences of its Fejér sums values at the given set of points
}

\author{
Alexander Kachurovskii, Ivan Podvigin
}

\begin{abstract}
It is shown that a continuous $2 \pi$-periodic function is uniquely recovered (on the whole real line) by sequences of its Fejér sums values at the given finite set of points if and only if there exist two of these points with the distance between them incommensurable with $\pi$. And that full sets of Fejér integrals at any two different points always uniquely recover continuous absolutely Lebesgue integrable on the real line function.

Wherein known sequence of Fejér sums values at a fixed single point $x \in \mathbb{R}$ and full set of Fejér integrals at this point determines uniquely a function only in the class of continuous functions with an even shift by $x$.
\end{abstract}

Анотація. Показано, що неперервна $2 \pi$-періодична функція однозначно відновлюється (на всій прямій) за відомими послідовностями значень своїх сум Фейєра в заданому скінченному наборі точок тоді і тільки тоді, коли знайдуться дві точки з цього набору, відстань між якими не $є$ співвимірними з $\pi$. Більш того, повні набори інтегралів Фейєра в будьяких двох різних точках завжди однозначно відновлюють неперервну функцію яка абсолютно інтегровна за Лебегом на всій числовій прямій.

При цьому відома послідовність значень сум Фейєра в одній фіксованій точці $x \in \mathbb{R}$ та повний набір інтегралів Фейєра в ній визначає однозначно функцію лише в класі таких неперервних функцій, зсув яких на $x \in$ парним.

The work was carried out in the framework of the State Contract of the Sobolev Institute of Mathematics (Project 0314-2019-0005)

2010 Mathematics Subject Classification: 42A16; 40G05

UDC 517.518.45+517.518.5

Keywords: Fejér sums, continuous $2 \pi$-periodic functions, Fejér integrals, continuous absolutely Lebesgue integrable on the real line functions, uniquely recovering

DOI: http://dx.doi.org/10.15673/tmgc.v13i3.1757 


\section{INTRODUCTION}

Let $L_{2 \pi}$ be the set of all $2 \pi$-periodic Lebesgue integrable on $(-\pi, \pi]$ functions $\rho: \mathbb{R} \rightarrow \mathbb{R}$. For every $\rho \in L_{2 \pi}$ we consider its Fejér sums

$$
\sigma_{n}(x)=\frac{1}{n} \sum_{k=0}^{n-1} S_{k}(x)=\sum_{|k|<n}\left(1-\frac{|k|}{n}\right) c_{k} e^{i k x}
$$

at every point $x \in \mathbb{R}$, where $S_{k}(x)$ and $c_{k}$ are the partial sums of the Fourier series of the function $\rho$ at the point $x$ and its Fourier coefficients (in the complex form), i.e.,

$$
S_{n}(x)=\sum_{|k| \leqslant n} c_{k} e^{i k x}, n \geqslant 0, \quad c_{k}=\frac{1}{2 \pi} \int_{-\pi}^{\pi} \rho(t) e^{-i k t} d t, k \in \mathbb{Z} .
$$

Further, let $C_{2 \pi}$ be the set of all $2 \pi$-periodic continuous functions $\rho: \mathbb{R} \rightarrow \mathbb{R}$. It is well known (see [2, Chapter VIII, §2] for example), that for any $\rho \in C_{2 \pi}$ its Fejér sums $\sigma_{n}(x)$ converge to $\rho(x)$ uniformly in $x$ as $n \rightarrow \infty$. Therefore, if the sequence of Fejér sums values $\left\{\sigma_{n}(x)\right\}_{n \geqslant 1}$ of continuous function is known for all points $x \in(-\pi, \pi]$ (or though for the points from some dense subset), then taking the limit as $n \rightarrow \infty$, we can easily recover this function.

In this note, we give an answer to a following question: is it possible to recover a function from $C_{2 \pi}$ if the sequences of its Fejér sums values $\left\{\sigma_{n}(x)\right\}_{n \geqslant 1}$ are known at the given set of points? Since (ibid) every continuous function is uniquely determined by its Fourier coefficients sequence $\left\{c_{k}\right\}_{k=-\infty}^{\infty}$, then the question is equivalent to the problem of uniquely recovering of these coefficients by the considered sequences of Fejér sums values.

It turns out that any such a function is uniquely recovered by the sequences of Fejér sums values which are known at any given (not necessary dense) infinite subset of points in $(-\pi, \pi]$. The sequence of Fejér sums values at any single point never can uniquely determine a function from $C_{2 \pi}$ : some additional information is needed; and sometimes, sequences of Fejér sums values at two different points are enough for a recovering of initial function.

Also, we solve similar problems for continuous absolutely Lebesgue integrable on the real line functions, with the replacing Fourier and Fejér sums with Fourier and Fejér integrals.

Some of the results under consideration were announced in [1].

\section{Fejér Sums}

2.1. Single fixed point. Note that a sequence of Fejér sums values of a function $\rho \in L_{2 \pi}$ at a point alows us immediately determine the sequence 
of partial Fourier sums at this point, because

$$
S_{0}(x)=\sigma_{1}(x), \quad S_{n}(x)=(n+1) \sigma_{n+1}(x)-n \sigma_{n}(x), n \geqslant 1 .
$$

We shall need obvious relations

$$
S_{0}(x)=c_{0}, \quad S_{n}(x)-S_{n-1}(x)=2 \operatorname{Re}\left(c_{n} e^{i n x}\right), n \geqslant 1,
$$

which are equivalent to relations

$$
\begin{gathered}
\sigma_{1}(x)=c_{0}, \quad \sigma_{2}(x)-\sigma_{1}(x)=\operatorname{Re}\left(c_{1} e^{i x}\right), \\
(n+1) \sigma_{n+1}(x)-2 n \sigma_{n}(x)+(n-1) \sigma_{n-1}(x)=2 \operatorname{Re}\left(c_{n} e^{i n x}\right), n \geqslant 2 .
\end{gathered}
$$

The following lemma shows, that the sequence of Fejér sums values at a given fixed single point $x$ can not recover uniquely an initial function from $C_{2 \pi}$.

Lemma 2.2. Given a function $\varphi \in L_{2 \pi}$, if its shift by $x$, i.e., the function $\varphi_{x}(t)=\varphi(x+t)$, is an odd function, then for every $\rho \in L_{2 \pi}$ the functions $\rho$ and $\rho+\varphi$ have the same sequences of Fejér sums (and partial Fourier sums also) values at the point $x$. For the case of continuous functions, the converse is true also, i.e., if two functions $\rho, \varrho \in C_{2 \pi}$ have the same sequences of Fejér sums values at the point $x$, then $\varphi_{x}=\rho_{x}-\varrho_{x}$ is an odd function.

Proof. As it follows from relations (2.2), for the direct statement it is enough to prove that for functions $\rho$ and $\rho+\varphi$ the numbers $\left\{2 \operatorname{Re}\left(c_{n} e^{i n x}\right)\right\}_{n=1}^{\infty}$ coincide. Note for this, that every such a number $2 \operatorname{Re}\left(c_{n} e^{i n x}\right)$ is a Fourier coefficient (in the real form) at cos $n t$ for the even part of the function $\rho_{x}(t)$ (which coincides with the even part of the function $\rho_{x}(t)+\varphi_{x}(t)$ ). But every even function has all the coefficients at $\sin n t$ equal 0 . Indeed,

$$
\begin{gathered}
\frac{1}{\pi} \int_{-\pi}^{\pi} \frac{\rho_{x}(t)+\rho_{x}(-t)}{2} \cos n t d t=\frac{1}{2 \pi} \int_{-\pi}^{\pi} \rho_{x}(t) \cos n t d t+ \\
+\frac{1}{2 \pi} \int_{-\pi}^{\pi} \rho_{x}(-t) \cos n t d t=\frac{1}{2 \pi} \int_{-\pi}^{\pi} \rho_{x}(t)\left(e^{i n t}+e^{-i n t}\right) d t= \\
=\frac{e^{-i n x}}{2 \pi} \int_{-\pi}^{\pi} \rho(t) e^{i n t} d t+\frac{e^{i n x}}{2 \pi} \int_{-\pi}^{\pi} \rho(t) e^{-i n t} d t= \\
=c_{-n} e^{-i n x}+c_{n} e^{i n x}=2 \operatorname{Re}\left(c_{n} e^{i n x}\right) .
\end{gathered}
$$

The converse statement of the lemma follows from such a note: the function $\varphi=\rho-\varrho$ at a point $x$ has zero sequence of Fejér sums; and so, taking (2.2) into account, it has zero coefficients $2 \operatorname{Re}\left(c_{n} e^{i n x}\right)$ also. As they are Fourier coefficients of the even part of continuous function $\varphi_{x}$, this 
(continuous also) even part is 0 identically. And so, $\varphi_{x}(t)=-\varphi_{x}(-t)$ for all $t \in \mathbb{R}$.

Remark 2.3. As Lemma 2.2 shows, the sequence of Fejér sums $\left\{\sigma_{n}(x)\right\}_{n=1}^{\infty}$ of continuous function $\rho$ at some point $x \in \mathbb{R}$ determines uniquely only the even part of the shift by $x$ of this function, i.e., the function

$$
\frac{\rho(t+x)+\rho(-t+x)}{2} \text {. }
$$

In other words, it means that the sequence of Fejér sums $\left\{\sigma_{n}(x)\right\}_{n=1}^{\infty}$ of function $\rho \in C_{2 \pi}$ at the fixed point $x \in \mathbb{R}$ determines uniquely function $\rho$ only in the class of functions from $C_{2 \pi}$ for which their shift by $x$ is even.

\section{TwO FIXED POINTS}

As we have seen from the previous considerations, continuous function generally could not be recovered by the sequence of its Fejér sums values at a single fixed point. Consider the case of two points.

Lemma 3.1. Every function $\rho \in L_{2 \pi}$ is uniquely determined (up to its values on the set of zero Lebesgue measure) by the sequences of its Fejér sums values $\left\{\sigma_{n}(x)\right\}_{n=1}^{\infty}$ and $\left\{\sigma_{n}(y)\right\}_{n=1}^{\infty}$ at any two points $x, y \in \mathbb{R}$ whose difference is incommensurable with $\pi$.

Proof. Assume that $x-y$ is incommensurable with $\pi$, and $c_{n}=a_{n}+i b_{n}$ are Fourier coefficients (in the complex form) of the function $\rho$. Then, taking into account relations (2.1), for every $n \geqslant 1$ we obtain the following system of two linear equations for coefficients $a_{n}$ and $b_{n}$ :

$$
\left\{\begin{array}{l}
S_{n}(x)-S_{n-1}(x)=2\left(a_{n} \cos n x-b_{n} \sin n x\right) \\
S_{n}(y)-S_{n-1}(y)=2\left(a_{n} \cos n y-b_{n} \sin n y\right) .
\end{array}\right.
$$

The determinant of the matrix of the system is equal to $2 \sin n(x-y)$, and it does not vanish by the condition on the difference $x-y$. Hence, all the coefficients $c_{k}=a_{k}+i b_{k}$ could be uniquely recovered; but it is well known (see [2, Chapter VIII, §2] for example), that a full set of Fourier coefficients determines function $\rho$ uniquely (up to its values on the set of zero Lebesgue measure).

Theorem 3.2. A function $\rho \in C_{2 \pi}$ is uniquely recovered (on the whole real line) by the sequences of its Fejér sums values $\left\{\sigma_{n}(x)\right\}_{n=1}^{\infty}$ and $\left\{\sigma_{n}(y)\right\}_{n=1}^{\infty}$ at any two fixed points $x, y \in \mathbb{R}$ if and only if $x-y$ is incommensurable with $\pi$. 
Proof. In the case when $x-y$ is incommensurable with $\pi$, the considered two sequences of Fejér sums values determine the initial function uniquely; it follows immediately from Lemma 3.1.

Consider now the case when $|x-y|=\frac{q}{p} \pi$ for some $q, p \in \mathbb{N}$. Then by Lemma 2.2, the continuous function $\rho(t)+\sin p(t-x)+(-1)^{q} \sin p(t-y)$ has the same sequences of Fejér sums values at points $x$ and $y$ as function $\rho$ has. And it is enough to make sure that the shifts by $x$ and by $y$ of the function

$$
\varphi(t)=\sin p(t-x)+(-1)^{q} \sin p(t-y)
$$

are odd functions. But this fact follows from the equality

$$
\sin p(t-x)=(-1)^{q} \sin p(t-y) .
$$

And so, the initial function can not be recovered uniquely in this case.

Remark 3.3. If the difference $x-y$ is commensurable with $\pi$, then the sequences of Fejér sums values $\left\{\sigma_{n}(x)\right\}_{n=1}^{\infty}$ and $\left\{\sigma_{n}(y)\right\}_{n=1}^{\infty}$ determine continuous function $\rho$ uniquely only in the class of functions from $C_{2 \pi}$ for which their shift by $x$ or by $y$ is an even function.

Remark 3.4. The sequences of Fejér sums values $\left\{\sigma_{n}\left(x_{j}\right)\right\}_{n=1}^{\infty}$ at the finite set of points $\left\{x_{j}\right\}_{j=1}^{m}, m \geqslant 3$ recover uniquely an initial function from $C_{2 \pi}$ if and only if there exists a couple of points from this set with the distance between them incommensurable with $\pi$.

Indeed, if such a couple of points exists, then the statement follows immediately from Theorem 3.2. In the case when pairwise distances between any two points from the set $\left\{x_{j}\right\}_{j=1}^{m}$ are commensurable with $\pi$, i.e., $\left|x_{i}-x_{j}\right|=\frac{q_{i j}}{p_{i j}} \pi$ with $i \neq j$ for $q_{i j}, p_{i j} \in \mathbb{N}$, then besides initial function $\rho(t)$ there exist additional functions with the same Fejér sums at all the points $\left\{x_{j}\right\}_{j=1}^{m}$. For example, the function $\rho(t)+\sin p\left(t-x_{1}\right)$, where $p=\prod_{j=2}^{m} p_{1 j}$, will be suitable. It is a consequence of Lemma 2.2 , because the shifts of the function $\sin p\left(t-x_{1}\right)$ by $x_{1}$ and by all $x_{j}$ with $j \geqslant 2$ are odd functions, since in the latest case

$$
\sin p\left(t-x_{1}\right)=(-1)^{\frac{p q_{1 j}}{p_{1 j}}} \sin p\left(t-x_{j}\right) .
$$

Infinite set of fixed points. It turns out that for any infinite set of points from $(-\pi, \pi]$ every continuous function can be recovered uniquely when the sequences of its Fejér sums values are known at these points.

Lemma 3.5. Let $X \subseteq(-\pi, \pi]$ be an infinite set of points, and for the function $\rho \in C_{2 \pi}$ its shifts by $x$, i.e., functions $\rho_{x}(t)=\rho(x+t)$, are odd for every $x \in X$. Then $\rho \equiv 0$. 
Proof. It is enough to prove that the set of zeros for continuous function $\rho$ is dense in $(-\pi, \pi]$, i.e., for any $\varepsilon>0$ there will be a finite $\varepsilon$-net, consisting from these zeros. In particular, the set of these zeros will contain all the points $x \in X$ since they are abscissas for centers of symmetry of the graph of the function $\rho$.

Fix any $\varepsilon>0$. Since the set $X \subseteq(-\pi, \pi]$ is infinite, then there exists a point $x \in X$ such that $x+\varepsilon_{0} \in X$ for some $\varepsilon_{0} \in(0, \varepsilon)$. But then $x+2 \varepsilon_{0}$, and $x+k \varepsilon_{0}$ for all $k \in \mathbb{Z}$ also are the abscissas for centers of symmetry of the graph of the function $\rho$; and so, they are zeros of this function as well. This proves the existence of the finite $\varepsilon$-net in $(-\pi, \pi]$ consisting from zeros of function $\rho$.

Theorem 3.6. Every function $\rho \in C_{2 \pi}$ is uniquely recovered by the sequences of its Fejér sums values $\left\{\sigma_{n}(x)\right\}_{n \in \mathbb{N}}$ at any infinite set of points $x$ from $(-\pi, \pi]$.

Proof. Suppose there is one more function $\varrho \in C_{2 \pi}$ with the same sequences of Fejér sums values at the given points $x$. Then by Lemma 2.2, every shift by $x$ of the difference $\rho-\varrho$, i.e., the function $\rho_{x}-\varrho_{x}$, will be an odd function. By Lemma 3.5, this difference is identically zero, i.e., $\rho \equiv \varrho$.

Remark 3.7. As it was noted by the reviewer, the method used in the proof of our key statements allows us to consider more general methods than Fejer's method (i.e., Chezaro summation), for example, Voronoi summation method.

\section{Fejér Integrals}

4.1. Single fixed point. Suppose now that $\rho: \mathbb{R} \rightarrow \mathbb{R}$ be absolutely Lebesgue integrable on whole real line function, i.e., $\rho \in L_{1}(\mathbb{R})$. At every point $x \in \mathbb{R}$ let us consider its Fejér integrals $\sigma_{t}(x)$ (continuous Chezaro averages for its Fourier integrals):

$$
\sigma_{t}(x)=\frac{1}{t} \int_{0}^{t} S_{\tau}(x) d \tau=\int_{-t}^{t}\left(1-\frac{|\tau|}{t}\right) e^{i \tau x} c_{\tau} d \tau
$$

(see, for example, formula (1.16.1) in [4, Chapter I, Section 1.16]); here $S_{t}(x)$ and $c_{t}$ are its Fourier integral and Fourier transform, respectively, i.e.,

$$
S_{t}(x)=\int_{-t}^{t} c_{\tau} e^{i x \tau} d \tau, \quad c_{t}=\frac{1}{2 \pi} \int_{\mathbb{R}} \rho(y) e^{-i t y} d y, \quad t \in \mathbb{R} .
$$

The properties of Fejér integrals of functions in question are analogous to the properties of Fejér sums of periodic functions. For example, it is well 
known (Theorem 14 in [4, Chapter I, §1.16]: G.H. Hardy, 1912), that in the case of continuity of function $\rho \in L_{1}(\mathbb{R})$ its Fejér integrals $\sigma_{t}(x)$ converge pointwise everywhere to $\rho(x)$ as $t \rightarrow \infty$ (uniformly in $x$ when $\rho$ is uniformly continuous: see [3, Chapter VII, §2]).

It is well known also (see, for example, [2, Chapter VIII, §4, Section 2]), that Fourier transform $c_{t}$ of function $\rho \in L_{1}(\mathbb{R})$ is (uniformly) continuous and bounded function; hence, for every $x \in \mathbb{R}$ mappings $S_{t}(x)$ and $\sigma_{t}(x)$ are continuous also. Besides, as it is easy to see, for all $x, t \in \mathbb{R}$ the following relations hold:

$$
S_{t}(x)=\lim _{\varepsilon \rightarrow 0+} \frac{1}{\varepsilon}\left((t+\varepsilon) \sigma_{t+\varepsilon}(x)-t \sigma_{t}(x)\right) .
$$

We also have the following counterparts of formulas (2.1) and (2.2):

$$
\begin{aligned}
& 2 \operatorname{Re}\left(c_{t} e^{i x t}\right)=\lim _{\varepsilon \rightarrow 0+} \frac{1}{\varepsilon}\left(S_{t+\varepsilon}(x)-S_{t}(x)\right)=\lim _{\varepsilon \rightarrow 0+} \frac{1}{\varepsilon}\left(S_{t}(x)-S_{t-\varepsilon}(x)\right), \\
& 2 \operatorname{Re}\left(c_{t} e^{i x t}\right)=\lim _{\varepsilon \rightarrow 0+} \frac{1}{\varepsilon^{2}}\left((t+\varepsilon) \sigma_{t+\varepsilon}(x)-2 t \sigma_{t}(x)+(t-\varepsilon) \sigma_{t-\varepsilon}(x)\right) .
\end{aligned}
$$

As in the case of Fejér sums considered in $§ 2$, a full set of Fejér integrals at a single point $x \in \mathbb{R}$ (or, equivalently, the complete set of integrals at this point) generally speaking does not determine uniquely continuous function $\rho \in L_{1}(\mathbb{R})$ : it is possible to recover from these data only the even part of its shift by $x$. Namely, the following statement is true (the analogue of Lemma 2.2 from $\S 2$ ).

Lemma 4.2. Suppose a function $\varphi \in L_{1}(\mathbb{R})$ is such that its shift by $x$, i.e., the function $\varphi_{x}(t)=\varphi(x+t)$, is an odd function. Then for every $\rho \in L_{1}(\mathbb{R})$ both functions $\rho$ and $\rho+\varphi$ have the same sets of Fejér integrals (and Fourier integrals also) at the point $x$.

Proof. From the definition of the Fejér and Fourier integrals, it can be seen that their values at the point $x$ are determined by the set $\left\{2 \operatorname{Re}\left(c_{t} e^{i t x}\right)\right\}_{t \in \mathbb{R}}$. Coefficient $2 \operatorname{Re}\left(c_{t} e^{i t x}\right)$ up to a factor is nothing but the cosine Fourier transform of the even part of the shift by $x$ of function $\rho(t)$ (which coincides with the even part of function $\left.\rho_{x}(t)+\varphi_{x}(t)\right)$. Indeed,

$$
\begin{aligned}
\frac{1}{\pi} \int_{\mathbb{R}} \frac{\rho_{x}(\tau)+\rho_{x}(-\tau)}{2} \cos t \tau d \tau= & \\
= & \frac{1}{\pi} \int_{\mathbb{R}} \rho_{x}(\tau) \cos t \tau d \tau=\frac{1}{2 \pi} \int_{\mathbb{R}} \rho_{x}(\tau)\left(e^{i t \tau}+e^{-i t \tau}\right) d \tau= \\
= & \frac{e^{-i t x}}{2 \pi} \int_{\mathbb{R}} \rho(\tau) e^{i t \tau} d \tau+\frac{e^{i t x}}{2 \pi} \int_{\mathbb{R}} \rho(\tau) e^{-i t \tau} d \tau=
\end{aligned}
$$




$$
=c_{-t} e^{-i t x}+c_{t} e^{i t x}=2 \operatorname{Re}\left(c_{t} e^{i t x}\right) .
$$

4.3. Two fixed points. As in the case of Fejér sums considered in $\S 2$, the complete sets of Fejér integrals known at two different points $x$ and $y$, already make it possible to recover the continuous function $\rho \in L_{1}(\mathbb{R})$. Moreover, no restrictions on $x-y$ are required here (therefore, we do not consider further the case of a collection of more than two fixed points in contrast to this case for Fejér sums in $\S 2$ ).

Lemma 4.4. Every function $\rho \in L_{1}(\mathbb{R})$ is uniquely recovered (up to values on a set of zero Lebesgue measure) by full collections of its Fejér integrals $\left\{\sigma_{t}(x)\right\}_{t \in \mathbb{R}}$ and $\left\{\sigma_{t}(y)\right\}_{t \in \mathbb{R}}$ at any two different points $x, y \in \mathbb{R}$.

Proof. It is seen from formula (4.1) that, by the known complete collections of Fejér integrals $\left\{\sigma_{t}(x)\right\}_{t \in \mathbb{R}}$ and $\left\{\sigma_{t}(y)\right\}_{t \in \mathbb{R}}$, we can recover $\operatorname{Re}\left(c_{t} e^{i x t}\right)$ and $\operatorname{Re}\left(c_{t} e^{i y t}\right)$ for all $t \in \mathbb{R}$.

Let $c_{t}=a_{t}+i b_{t}$ and

$$
C_{t}(z)=\lim _{\varepsilon \rightarrow 0+} \frac{1}{2 \varepsilon^{2}}\left((t+\varepsilon) \sigma_{t+\varepsilon}(z)-2 t \sigma_{t}(z)+(t-\varepsilon) \sigma_{t-\varepsilon}(z)\right), z \in \mathbb{R} .
$$

Then for every $t \in \mathbb{R}$ we obtain the following system of two linear equations for coefficients $a_{t}, b_{t}$ :

$$
\left\{\begin{array}{l}
a_{t} \cos t x-b_{t} \sin t x=C_{t}(x) \\
a_{t} \cos t y-b_{t} \sin t y=C_{t}(y) .
\end{array}\right.
$$

The determinant of the matrix of the system is $\sin t(x-y)$.

Consider two cases. If $x-y$ is incommensurable with $\pi$, then for all $t \in \mathbb{Q}$ the determinant does not vanish; hence, the Fourier transform $c_{t}$ for such $t$ is uniquely recovered.

And if $x-y$ is commensurable with $\pi$, then for all $t \in \xi \mathbb{Q}$, where $\xi$ is any real number incommensurable with $\pi$, the determinant also does not vanish, and the Fourier transform $c_{t}$ is recovered at such points $t$.

In any case, we can recover the Fourier transform on a dense subset; it is recovered by continuity on the whole real line. Moreover, the Fourier transform $c_{t}$ a.e. uniquely determines function $\rho \in L_{1}(\mathbb{R})$. It is a consequence of a well known (see, for example, [2, Chapter VIII, §4, Section 1]) property of Fourier transform in $L_{1}(\mathbb{R})$ : if $c_{t} \equiv 0$ then $\rho=0$ a.e.

The following theorem is an immediate consequence of Lemma 4.4. 
Theorem 4.5. Every continuous function $\rho \in L_{1}(\mathbb{R})$ is uniquely recovered (on the whole line) by full collections of its Fejér integrals $\left\{\sigma_{t}(x)\right\}_{t \in \mathbb{R}}$ and $\left\{\sigma_{t}(y)\right\}_{t \in \mathbb{R}}$ at any two different points $x, y \in \mathbb{R}$.

Remark 4.6. Obviously, the statement of the theorem remains true if the complete collections of Fejér integrals are replaced by collections defined on dense subsets, i.e., $\left\{\sigma_{t}(x)\right\}_{t \in R_{1}}$ and $\left\{\sigma_{t}(y)\right\}_{t \in R_{2}}$, where $R_{1}$ and $R_{2}$ are dense in $\mathbb{R}$.

\section{REFERENCES}

[1] A. G. Kachurovskii, I. V. Podvigin. Fejér sums and Fourier coefficients of periodic measures. Doklady Mathematics, 98(2):464-467, September 2018, doi: $10.1134 / \mathrm{s} 1064562418060170$.

[2] A.N. Kolmogorov, S.V. Fomin. Elements of the theory of functions and functional analysis. Moscow: Nauka, 1976 (in Russian).

[3] G. Ye. Shilov. Mathematical analysis. A special course. Translated by J. D. Davis. English translation edited by D. A. R. Wallace. Pergamon Press, Oxford-New York-Paris, 1965.

[4] E. C. Titchmarsh. Introduction to the theory of Fourier integrals. Chelsea Publishing Co., New York, third edition, 1986.

Received: May 20, 2020, accepted: July 29, 2020.

Alexander Kachurovskii

Sobolev Institute of Mathematics, Novosibirsk, Russia

Email: agk@math.nsc.ru

ORCID: orcid.org/0000-0002-2747-2660

Ivan Podvigin

Sobolev Institute of Mathematics, Novosibirsk, Russia; Novosibirsk State UniVERsity, Novosibirsk, Russia

Email: ipodvigin@math.nsc.ru 